PAEDIATRIC LUNG DISEASE

\title{
Clinical features, aetiology and outcome of empyema in children in the north east of England
}

\author{
K M Eastham, R Freeman, A M Kearns, G Eltringham, J Clark, J Leeming, D A Spencer
}

Thorax 2004;59:522-525. doi: 10.1136/thx.2003.016105

See end of article for authors' affiliations ......................

Correspondence to: Dr D A Spencer, Department of Respiratory Paediatrics, Freeman Hospital, Newcastle upon Tyne NE7 7DN, UK; david. spencer@nuth.northy.nhs. uk

Received

21 September 2003

Accepted

20 February 2004
Background: The incidence of empyema in children in the UK is increasing. The reason for this is unclear. A prospective study was undertaken to investigate the clinical features, aetiology, and outcome of cases of empyema and parapneumonic effusion presenting to a tertiary paediatric respiratory centre between February 1997 and August 2001.

Method: Routine bacterial culture of blood and pleural fluid was performed for 47 cases. Forty three pleural fluid specimens, culture negative for pneumococcus, were analysed for pneumococccal DNA by real time polymerase chain reaction (PCR). Penicillin susceptibility was determined for DNA positive specimens using complementary PCR assay. Capsular serotype specific antigen detection was by enzyme immunoassay (EIA) using monoclonal antibodies to serotypes 1, 3, 4, 5, 6A, 6B, 7F, 9V, 14, 18C, 19A, $19 \mathrm{~F}$, and $23 \mathrm{~F}$. Clinical data were obtained from patient notes, supplemented by a postal questionnaire. Results: The median (range) age of the patients was $5.6(0.6-16.9)$ years and $70 \%$ were male. The median (range) duration of illness before referral to hospital was 5 (0-25) days. Forty five (96\%) had received antibiotics before referral; $32(68 \%)$ required decortication and eight (21\%) thoracocentesis. Median postoperative stay was 4 days (2-8). Thirty two (75\%) pneumococcal culture negative specimens were pneumococcal DNA positive; $17(53 \%)$ of these were serotype 1 . All were penicillin sensitive. Conclusions: Pneumococcus is the major pathogen in childhood empyema and serotype 1 is the prevalent serotype. This has implications for vaccine development and immunisation strategy as the current 7-valent pneumococcal conjugate vaccine does not protect against serotype 1.
$\mathrm{E}$ mpyema and parapneumonic effusion complicating pneumonia has increased dramatically in children in the UK over the last decade. ${ }^{2}$ An explanation for this phenomenon has yet to be determined. Understanding of the problem has been hampered by the fact that routine bacterial culture is negative in most cases, presumably because antibiotics have usually been administered before referral to the regional centre. Empyema and complex parapneumonic effusion represent parts of a spectrum of disease, with three stages of progression being recognised: ${ }^{3}$

- Stage 1 ("exudative"): fluid accumulation within the pleural cavity without the presence of loculations (parapneumonic effusion).

- Stage 2 ("fibropurulent"): fibrin deposition within the pleural space giving rise to loculations; presence of pus (empyema).

- Stage 3 ("organisational"): organised multiloculated empyema with lung entrapment and pleural rind formation.

Management at our centre is determined by clinical features aided by ultrasound findings, as described previously. ${ }^{5}$

Streptococcus pneumoniae has been found to be the principal pathogen in childhood empyema in the USA, with serotype 1 accounting for $24-50 \%$ of culture positive cases between 1993 and $2000 .{ }^{67}$ Clonal spread of this serotype has been proposed as a possible explanation. This is supported by the recent emergence of a novel clone of serotype 1 in Sweden which has been associated with a fourfold increase in invasive pneumococcal disease. ${ }^{8}$ In contrast, serotype 14 has been the most prevalent serotype causing invasive pneumococcal disease in England and Wales, although empyema related strains have not been specifically collected or typed.'
The purpose of this investigation was to describe the clinical features, aetiology, and outcome of empyema and complex parapneumonic effusion in children presenting to Freeman Hospital, a tertiary paediatric centre in the north east of England, and to investigate the role of $S$ pneumoniae using new microbiological techniques.

\section{METHOD}

Forty seven consecutive cases of empyema or complex parapneumonic effusion were recruited between February 1997 and August 2001. Referrals were all from other hospitals in the Northern Region and north Cumbria (Whitehaven and Carlisle). Informed parental consent was obtained. All cases were less than 17 years of age with clinical and radiological evidence of empyema or pleural effusion requiring management by thoracocentesis and/or decortication. Ultrasound examination staged the disease in 20 cases. The study was approved by the Newcastle and North Tyneside local research ethics committee.

A sample of $10 \mathrm{ml}$ pleural fluid was collected at the time of chest drain insertion. Routine bacterial culture (including methods for anaerobes) and latex agglutination tests for pneumococcal antigen (SLIDEX, BioMérieux, France) were performed in all cases. In 43 cases sufficient pleural fluid was available for further analysis. A real time polymerase chain reaction (PCR) assay was performed for the detection of pneumococcal DNA. ${ }^{10}$ Pneumococcal DNA positive samples underwent a second complementary PCR assay targeting a conserved sequence of the $p b p 2 b$ gene which is found in all strains of penicillin susceptible pneumococci (minimum inhibitory concentration to penicillin $<0.06 \mathrm{mg} / \mathrm{l}) .{ }^{11}$ A solid phase indirect sandwich enzyme linked immunosorbent assay (ELISA) was performed on the 43 specimens to detect type specific pneumococcal capsular polysaccharide using 
monoclonal antibodies against types $1,3,4,5,6 \mathrm{~A}, 6 \mathrm{~B}, 7 \mathrm{~F}, 9 \mathrm{~V}$, 14, 18C, 19A, 19F and 23F (donated by Wyeth Vaccines).

Demographic and clinical data included age, sex, past medical history, duration of illness at presentation and referral, microbiological and radiological investigations, antibiotic use, length of hospital stay, and clinical outcome. Spirometric tests were performed at least 1 year after decortication in 11 of 16 children aged more than 5 years in May 2001. Data were collected by a postal questionnaire issued in October 2001 (3-56 months after admission to hospital) on work or school absence of family members during the acute illness.

\section{RESULTS}

\section{Clinical features}

Figure 1 illustrates case frequency, and the age distribution of the 47 children is shown in fig 2 . The median age was 5.6 years (range 0.6-16.9); the male:female ratio was 2:1. Thirty one of the children $(66 \%)$ had been previously fit and well; four had a concurrent infection at time of presentation (three varicella zoster and one Campylobacter gastroenteritis); eight had experienced a significant previous respiratory morbidity (three bronchiolitis, three recurrent respiratory tract infection, two asthma); two had a haematological malignancy and were undergoing chemotherapy; and two children had a chronic neurological disability with impaired mobility (spastic quadriplegia and metachromatic leucodystrophy).

The median duration of illness before admission to hospital was 5 days (range 0-25) and the median primary hospital stay was 3 days (range 0-14). Forty five cases (96\%) had received antibiotics before transfer; 29 (61\%) had received an antibiotic combination which included a cephalosporin and $22(47 \%)$ included a penicillin.

\section{Laboratory findings}

A pathogen was implicated in 36 of the 47 cases (31 S pneumoniae; one dual infection with $S$ pneumoniae and cytomegalovirus; one Staphylococcus aureus; one Streptococcus pyogenes; one Streptococcus viridans; and one Mycobacterium tuberculosis). All children had a blood culture taken at Freeman Hospital and six were positive (five $S$ pneumoniae and one $S$ viridans). All five cases with pneumococcal positive blood cultures were subsequently shown to have DNA evidence of pneumococcal infection in the pleural fluid. Routine bacterial culture of pleural fluid was negative in 44 cases (94\%). Three specimens were culture positive: one Staphylococcus epidermidis which was considered to be a contaminant, one $S$ pyogenes, and one $S$ aureus in a child with a coexistent lung abscess. Mycobacterium tuberculosis (TB) was diagnosed by PCR in one child with an operative finding of pleural plaques in whom there was histological evidence of necrotising granulomata. Cytomegalovirus was isolated from viral culture of pleural fluid in a further child, performed because she had acute lymphoblastic leukaemia (ALL) and was undergoing chemotherapy.

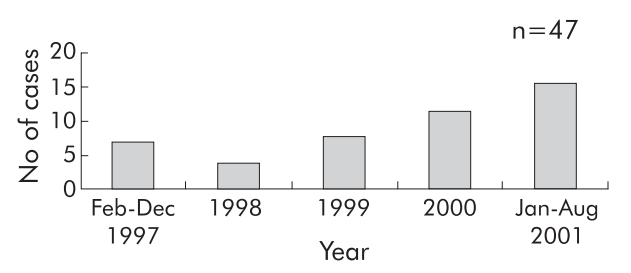

Figure 1 Number of cases of childhood empyema and parapneumonic effusion presenting to Freeman Hospital, February 1997 to August 2001.

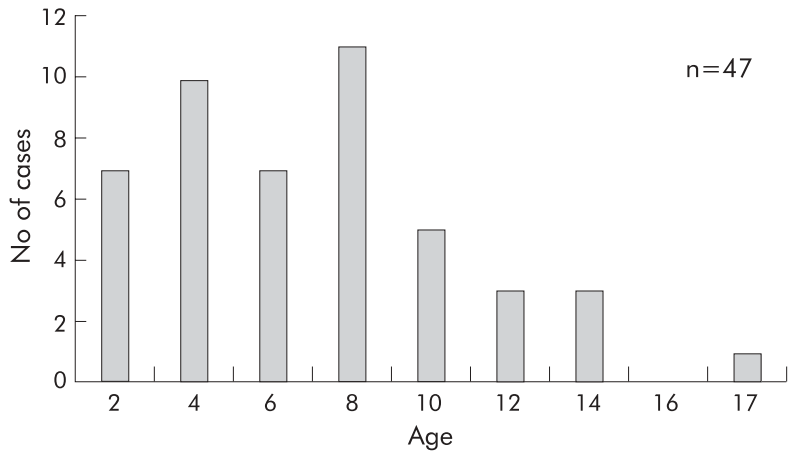

Figure 2 Age distribution of cases of empyema and parapneumonic effusion.

Pneumococcal latex agglutination antigen testing was positive in 12 of 47 cases, all accounted for on PCR. Sufficient pleural fluid was available for further analysis in 43 of the 47 cases (fig 3). All were negative for $S$ pneumoniae on routine culture. Pneumococcal DNA was identified by PCR in 32 cases $(75 \%)$, illustrating the poor sensitivity of pneumococcal latex agglutination testing relative to PCR. Additional molecular analysis showed the presence of the conserved sequence associated with penicillin susceptibility in all 32 specimens. Figure 4 shows the serotype distribution.

\section{Management}

Surgical management formed the mainstay of treatment. Unsuspected lung abscess was an operative finding in seven cases. Pneumococcus serotype 1 was confirmed in three cases, serotype 14 in two cases, and serotype 3 in one case of lung abscess. Thirty two children $(68 \%)$ required decortication including four cases managed initially by thoracocentesis. A further eight patients required thoracocentesis alone. Three children were managed initially with intrapleural urokinase but this failed to effect clinical resolution in two patients in whom decortication was subsequently required.

The median postoperative time to defervescence was less than 24 hours (range 0-6 days). Median stay in the intensive care unit for the cohort was less than 24 hours (range 1-48) and median postoperative inpatient stay was 4 days (range 2-8). Five children were found to be anaemic, three of whom were given a blood transfusion and two oral iron. Two children were found to have pre-renal failure secondary to hypovolaemia. Renal function was normal at discharge following appropriate fluid management. T cell lymphoblastic lymphoma was diagnosed in one child in whom a pleural effusion recurred 10 days after discharge.

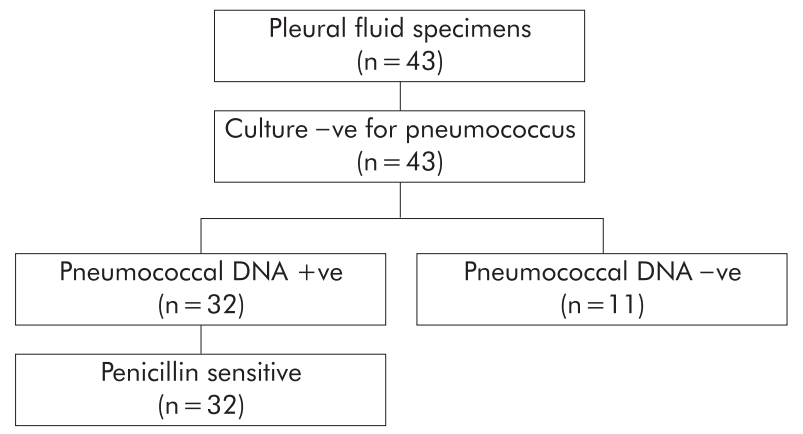

Figure 3 Detailed microbiological analysis of 43 pleural fluid specimens. 


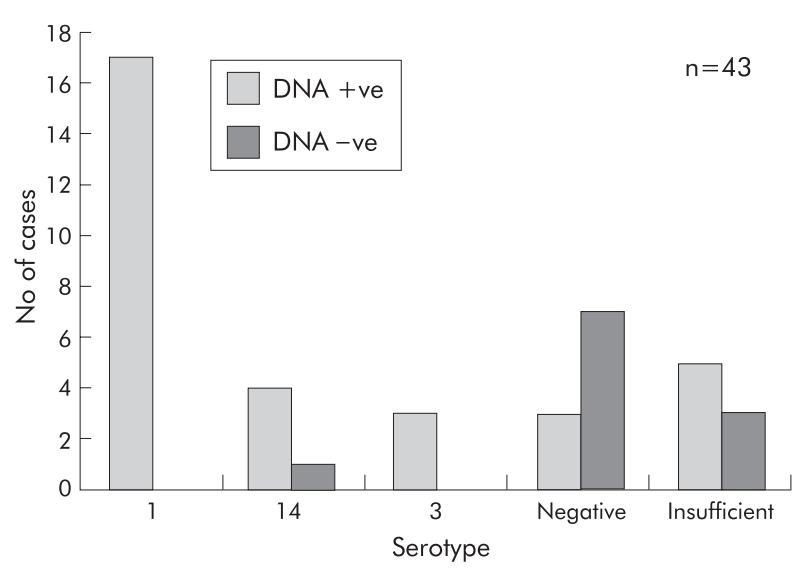

Figure 4 Pneumococcal serotype distribution of culture negative cases.

\section{Outcome}

The children were reviewed as outpatients 1 month after discharge. Forty one $(87 \%)$ children were clinically well, three were undergoing chemotherapy for haematological malignancy, two had experienced persistent cough, and one persistent cough and breathlessness with exercise. Seven children had low specific antibody titres in convalescence (one pneumococcus, Haemophilus influenzae B (Hib) and tetanus; two pneumococcus and Hib; one pneumococcus and tetanus; two pneumococcus ; one tetanus). One child had low $\operatorname{IgG}_{2}$. The median age of the six children with low pneumococcal titres was 2.4 years (range 1.6-6.4). Serotyping was performed in five of these cases, confirming that infection was due to pneumococcus (serotype 1 in three children, serotype 14 in one; and serotype 3 in one).

The parental questionnaire was returned in $60 \%$ of cases. The duration of time reported to have been missed from work for employed parents as a direct result of the child's illness, and missed from school or nursery school for children old enough to attend, was based on a 5 day week from the beginning of onset of symptoms. A median of 10 working days (range 1-30) were lost for 17 fathers, 30 working days (range 4-75) lost for 15 mothers, and 30 school or nursery school days (range 10-75) missed by 20 children.

Lung function tests were repeated in 11 of 16 children aged more than 5 years at least 1 year following decortication. Ten had reproducible flow-volume loops. The median forced expiratory volume in 1 second $\left(\mathrm{FEV}_{1}\right)$ was $85 \%$ predicted (range 70-108), median forced vital capacity (FVC) was $90 \%$ predicted (range 64-100), and median $\mathrm{FEV}_{\mathrm{l}} / \mathrm{FVC} 84 \%$ predicted (range 71-100) according to a standard reference range. ${ }^{12} \mathrm{~A}$ diagnosis of asthma was made in the three children with an obstructive defect, all of whom had evidence of reversibility with a bronchodilator.

\section{DISCUSSION}

An increase in the incidence of empyema in the UK has been reported since 1995, but the aetiology has been largely unknown. ${ }^{12}$ A similar trend in certain geographical areas of the USA has been attributed to pneumococcal serotype 1 in culture positive cases. ${ }^{67}$

The tertiary referral system in the UK differs from the USA where children present directly to the hospital for management of their empyema. In our series the median time before presentation to hospital was 5 days and $96 \%$ of children had received antibiotics before referral to our centre. It is therefore not surprising that routine culture of pleural fluid and blood was negative in most cases. This study benefits from the investigation of pneumococcal aetiology and serotype in culture negative cases. It parallels the culture positive US experience and thus confirms the role of $S$ pneumoniae as the principal pathogen in childhood empyema in the UK, with type 1 being the dominant serotype.

Most of the children had no predisposing risk factors to infection. While parapneumonic effusion is the most common cause of a pleural effusion in children, it is important to consider other diagnoses as illustrated by our finding of a case of TB and another of haematological malignancy.

Surgical management was associated with a favourable outcome. The median postoperative stay was 4 days with defervesence within 24 hours; $87 \%$ of cases were well at 1 month follow up. Lung function tests were normal in seven children old enough to cooperate at least 1 year following decortication, and three children with an obstructive defect had coexistent asthma and evidence of reversibility with a bronchodilator.

We observed an increase in the number of cases of empyema and parapneumonic effusion in 2001 relative to previous years (fig 1), and this trend has continued with a further 28 cases presenting to Freeman Hospital between August 2001 and July 2003. It is important to speculate why this has occurred and why serotype 1 might be re-emerging with such virulence in western societies.

The median age of our series was 5.6 years. This is consistent with the US data $^{67}$ and suggests that either type 1 pneumococcus has increased virulence in childhood pneumonias or that a virulent clone of pneumococcus has emerged in the community. Serotypes 1, 2 and 3 were responsible for $75 \%$ of bacteraemia in the western world in the early 20th century. ${ }^{13} 14$ Serotype 1 is seen now most commonly in developing countries such as Rwanda and Eygpt. ${ }^{15}$ It has been suggested that poverty, overcrowding, and decreased availability of antibiotics have resulted in the increased prevalence of this serotype in the developing world, ${ }^{16}$ although this does not explain its emergence in the affluent state of Utah in the USA. ${ }^{7}$ Patients with pneumonia complicated by a pleural effusion are more likely to be older, of white race, and have chest pain on presentation than patients with uncomplicated pneumonia. ${ }^{6}$ We propose that serotype 1 pneumococci have a characteristic that facilitates persistence of the organism in infected tissue.

Pneumococcal vaccination has been offered in the routine immunisation schedule in the USA since the year $2000 .{ }^{17}$ The 7 -valent pneumococcal vaccine protects against serotypes 4, $6 \mathrm{~B}, 9 \mathrm{~V}, 14,18 \mathrm{C}, 19 \mathrm{~F}$, and $23 \mathrm{~F}$ but not serotype 1 . It could be argued that introduction of the vaccine has favoured the proliferation of novel clones not covered by it. However, this could not account for the observations made in US studies ${ }^{67}$ in patients recruited before the introduction of widespread vaccination or for our observations, as the 7-valent pneumococcal conjugate vaccine has only recently been introduced in high risk groups in the UK. ${ }^{18}$ Furthermore, the incidence of complicated community acquired pneumonia has been reported to have decreased since the introduction of the 7valent pneumococcal conjugate vaccine in the USA. ${ }^{19}$

Selection is unlikely to be driven by antibiotic resistance as all the strains are penicillin sensitive (cephalosporins were used most widely in our study population). We hypothesise that the phenomenon of antibiotic tolerance, whereby bacteria evade the killing action of the antibiotic without an increase in the minimum inhibitory concentration (MIC), may be a potential explanation for the re-emergence of serotype 1 in western societies..$^{20}$ Alternatively, the emerging serotype may be producing an as yet unrecognised factor important in its survival.

The factors responsible for the dominance of pneumococcal serotype 1 in childhood empyema in the UK remain to be determined. If antibiotic tolerance is found to be a 
pathophysiological mechanism, this would have widespread implications for antibiotic policy in the community and hospital management of lower respiratory tract infections. Although a recent analysis of the cost of pneumococcal disease in childhood suggests that childhood empyema does not constitute a significant economic burden, ${ }^{21}$ we suggest that the severity and increasing prevalence of this condition does support a strategy of routine pneumococcal vaccination in early childhood. This would require the introduction of next generation vaccines (9-valent) to ensure the full impact of vaccination as the new 7-valent pneumococcal conjugate vaccine, recently licensed for use, does not protect against serotype 1 . These findings have implications both for vaccine development and immunisation strategies in childhood.

\section{Authors' affiliations}

K M Eastham, Sir James Spence Institute of Child Health, Royal Victoria Infirmary, Newcastle upon Tyne NE1 4LP, UK

R Freeman, A M Kearns, G Eltringham, Public Health Laboratory,

Newcastle General Hospital, Newcastle upon Tyne NE4 6BE, UK

J Clark, Department of Paediatric Infectious Diseases, Newcastle

General Hospital, Newcastle upon Tyne NE4 6BE, UK

J Leeming, Public Health Laboratory, Bristol Royal Infirmary, Bristol BS2 $8 \mathrm{HW}, \mathrm{UK}$

D A Spencer, Department of Respiratory Paediatrics, Freeman Hospital, Newcastle upon Tyne NE7 7DN, UK

\section{REFERENCES}

1 Rees JH, Spencer DA, Parikh D, et al. Increase in incidence of childhood empyema in West Midlands, UK. Lancet 1997;349:402.

2 Playfor SD, Smyth AR, Stewart RJ. Increase in incidence of childhood empyema. Thorax 1997:52:932.

3 American Thoracic Society. Management of non-tuberculous empyema. Am Rev Respir Dis 1962;85:935-6.

4 Light RW. A new classification of parapneumonic effusions and empyema. Chest 1995; 108:299-301.

5 Carey JA, Hamilton JR, Spencer DA, et al. Empyema thoracis: a role for open thoracotomy and decortication. Arch Dis Child 1998;79:510-3.
6 Tan TQ, Mason EO Jr, Wald ER, et al. Clinical characteristics of children with complicated pneumonia caused by Streptococcus pneumoniae. Pediatrics 2002;110:1-6.

7 Byington CL, Spencer LY, Johnson TA, et al. An epidemiological investigation of a sustained high rate of pediatric parapneumonic empyema: risk factors and microbiological associations. Clin Infect Dis 2002;34:434-40.

8 Henriques Normark B, Kalin M, Ortqvist A, et al. Dynamics of penicillinsusceptible clones in invasive pneumococcal disease. J Infect Dis 2001;184:861-9.

9 Miller E, Waight P, Efstratiou A, et al. Epidemiology of invasive and other pneumococcal disease in children in England and Wales 1996-1998. Acto Paediatr Suppl 2000;89:11-6.

10 Kearns AM, Freeman R, Murphy OM, et al. Rapid PCR-based detection of Streptococcus pneumoniae DNA in cerebrospinal fluid. J Clin Microbiol 1999;37:343-4.

11 Kearns AM, Graham C, Burdess D, et al. Rapid real-time PCR for determination of penicillin susceptibility in pneumococcal meningitis, including culture-negative cases. J Clin Microbiol 2002;40:682-4.

12 Rosenthal M, Bain SH, Cramer D, et al. Lung function in white children aged 4 to 19 years: 1 -Spirometry. Thorax 1993;48:794-802.

13 Finland $M$, Barnes MW. Changes in occurrence of capsular serotypes of Streptococcus pneumoniae at Boston City Hospital during selected years between 1935 and 1974. J Clin Microbiol 1977;5:154-66.

14 Watson DA, Musher DM, Verhoef J. Pneumococcal virulence factors and host immune responses to them. Eur J Clin Microbiol Infect Dis 1995; 14:479-90

15 Greenwood BM, Hassan-King M, Onyemelukwe G, et al. Pneumococcal serotypes in West Africa. Lancet 1980;1:360.

16 Dagan R, Gradstein S, Belmaker I, et al. An outbreak of Streptococcus pneumoniae serotype 1 in a closed community in southern Israel. Clin Infect Dis 2000;30:319-21

17 American Academy of Pediatrics. Committee on Infectious Diseases. Policy statement: recommendations for the prevention of pneumococcal infections, including the use of pneumococcal conjugate vaccine (Prevnar), pneumococcal polysaccharide vaccine, and antibiotic prophylaxis. Pediatrics 2000;106:362-6.

18 Finn A, Booy R, Moxon R, et al. Should the new pneumococcal vaccine be used in high-risk children? Arch Dis Child 2002;87:18-21.

19 Buckingham SC, King MD, Miller ML. Incidence and etiologies of complicated parapneumonic effusions in children. Pediatr Infect Dis J 2003;22:499-504.

20 Tuomanen E, Durack DT, Tomasz A. Antibiotic tolerance among clinical isolates of bacteria. Antimicrob Agents Chemother 1986;30:521-7.

21 Mcintosh EDG, Conway P, Willingham J, et al. The cost-burden of pediatric pneumococcal disease in the UK and the potential cost-effectiveness of prevention using 7 -valent pneumococcal conjugate vaccine. Vaccine 2003;21:2564-72. 